Article

\title{
Short Chain $N$-Acylhomoserine Lactone Production by Clinical Multidrug Resistant Klebsiella pneumoniae Strain CSG20
}

\author{
Yun Fong Ngeow ${ }^{1}$, Huey Jia Cheng ${ }^{2}$, Jian Woon Chen ${ }^{2}$, Wai-Fong Yin ${ }^{2}$ and Kok-Gan Chan ${ }^{2, *}$ \\ 1 Department of Medical Microbiology, Faculty of Medicine, University of Malaya, \\ Kuala Lumpur 50603, Malaysia; E-Mail: yunngeow@um.edu.my \\ 2 Division of Genetics and Molecular Biology, Institute of Biological Sciences, Faculty of Science, \\ University of Malaya, Kuala Lumpur 50603, Malaysia; E-Mails: chenghj90@gmail.com (H.J.C.); \\ cjw246@hotmail.com (J.W.C.); yinwaifong@yahoo.com (W.-F.Y.)
}

* Author to whom correspondence should be addressed; E-Mail: kokgan@um.edu.my; Tel.: +603-7967-5162; Fax: +603-7967-4509.

Received: 20 September 2013; in revised form: 23 October 2013 / Accepted: 31 October 2013/ Published: 7 November 2013

\begin{abstract}
Klebsiella pneumoniae is one of the most common Gram-negative bacterial pathogens in clinical practice. It is associated with a wide range of disorders, ranging from superficial skin and soft tissue infections to potentially fatal sepsis in the lungs and blood stream. Quorum sensing, or bacterial cell-cell communication, refers to population density-dependent gene expression modulation. Quorum sensing in Proteobacteria relies on the production and sensing of signaling molecules which are mostly $\mathrm{N}$-acylhomoserine lactones. Here, we report the identification of a multidrug resistant clinical isolate, K. pneumoniae strain CSG20, using matrix-assisted laser desorption ionization-time-offlight (MALDI-TOF) mass spectrometry. We further confirmed quorum sensing activity in this strain with the use of high resolution tandem liquid chromatography quadrupole mass spectrometry and provided evidence $K$. pneumoniae strain CSG20 produced $\mathrm{N}$-hexanoyl-homoserine lactone (C6-HSL). To the best of our knowledge, this is the first report on the production of $N$-hexanoylhomoserine lactone (C6-HSL) in clinical isolate K. pneumoniae.
\end{abstract}

Keywords: Klebsiella pneumonia; multidrug resistant; mass spectrometry; MALDI-TOF; $\mathrm{N}$-hexanoylhomoserine lactone (C6-HSL); clinical pathogen; quorum sensing 


\section{Introduction}

Klebsiella pneumoniae is found ubiquitously in the natural environment and mammalian mucous membranes [1]. In humans, it forms part of the normal flora in the alimentary tract [2]. It is common hospital-acquired pathogen causing a variety of disorders, ranging from superficial wound infections to severe, life-threatening sepsis $[3,4]$. The most serious community-acquired infection is a fulminant pneumonia that is more common among chronic alcoholics, diabetics and older age groups with debilitating illnesses [5-7]. Risk factors for nosocomial infection or colonization include multiple antibiotic therapies, treatment in an intensive care unit and contamination of indwelling medical devices such as ventilator tubings, central venous and urinary catheters [8]. In the past few decades, extensive use of broad-spectrum antibiotics has led to the emergence of strains that show plasmid- or transposon-mediated resistance to multiple antibiotics including the $\beta$-lactam antibiotics, fluoroquinolones, aminoglycosides, cotrimoxazole and tetracyclines [9-12].

Quorum sensing (QS) typically refers to a process of bacterial cell-to-cell communication that relies on the production and detection of extracellular signaling molecules called autoinducers. In Gram-negative bacteria, autoinducers are mostly derivatives of an $N$-acylhomoserine lactone (AHL) backbone with species-specific substitutions [13]. QS allows bacterial cells to control gene expression collectively, and thus synchronize bacterial group behavior at a population density-dependent manner. QS-controlled phenotypic processes are unproductive unless a high density of bacterial cells acts in unison. The common goal of colonizing or infecting a host is a task that is often impossible for a single cell to achieve; a collective effort via the QS mechanism, however, will greatly enhance the chance of success. Many of the biological functions controlled by QS are associated with bacterial virulence, such as the production of extracellular polysaccharides [14], biofilm formation [15-17], iron binding and innate bactericidal activities [18]. On this basis, research in QS in clinical pathogens will allow a better understanding of virulence determinants production and the molecular basis of pathogenicity.

In this study, we examined the QS activity of a clinical isolate, $K$. pneumoniae strain CSG20, using a biosensor assay and high resolution tandem liquid chromatography quadrupole mass spectrometry for the detection and characterization of AHLs.

\section{Experimental Section}

\subsection{Bacterial Strains and Culture Conditions}

Strain CSG20 was isolated from the sacral wound swab of a patient who had undergone a craniotomy that led to a two-month post-operative recovery in the hospital. It was a non-motile, oxidase-negative and Gram-negative bacillus that grew as large, mucoid, lactose-fermenting colonies on Mac Conkey agar after $24 \mathrm{~h}$ incubation at $36^{\circ} \mathrm{C}$. Next, it was sub-cultured onto Tryptic Soy Agar (Scharlau, Barcelona, Spain) for bacterial identification. In the routine diagnostic laboratory, it was identified as K. pneumoniae by the IMVIC reactions (indole-negative, methyl red-positive, Voges Proskauer-negative and citrate-positive) and was found to be a producer of extended-spectrum $\beta$-lactamase (ESBL). The agar disk diffusion (Kirby-Bauer) test showed in vitro resistance to ampicillin, amoxicillin-clavulanate, ampicillin-sulbactam, cephalexin, cefuroxime and cotrimoxazole but susceptibility to sulperazone, imipenem, gentamicin, netilmicin and ciprofloxacin. 
Chromobacterium violaceum CV026 was used as the QS biosensor. This is a double mini-Tn5 mutant derived from ATCC31532 that produces violacein pigment only in the presence of $N$-acyl side chains of 4-8 carbons [19]. It was grown in Luria Bertani (LB) broth (1\% w/v tryptone, 1\% w/v sodium chloride and $0.5 \% \mathrm{w} / \mathrm{v}$ yeast extract), or LB agar (LBA) (LB with addition of $1.5 \% \mathrm{w} / \mathrm{v}$ Bacto-agar) incubated at $28{ }^{\circ} \mathrm{C}$.

\subsection{Sample Preparation for MALDI-TOF MS and Data Analysis.}

A single colony of overnight culture strain CSG20 was smeared onto the MSP 96 target polished steel BC plate and subjected to MALDI-TOF MS analysis as reported previously [20]. It was analyzing using Microflex MALDI-TOF (Bruker Daltonik GmbH, Leipzig, Germany) bench-top mass spectrometer (equipped with UV laser at wavelength $337 \mathrm{~nm}$ ) with the Bruker FlexControl software Version 3.3 (Build 108). The spectra were recorded in the linear positive ion mode and analyzed over a mass range of 2 to $20 \mathrm{kDa}$ (acceleration voltage set at $20 \mathrm{kV}$ ). MALDI-TOF spot on the target plate was measured by the MBT-autoX.axe autoExecute method resulted from six series of 40 laser shots at different positions on the spotted product. The MALDI-TOF spectra were then analyzed in the Bruker MALDI Biotyper Real Time Classification (RTC) Version 3.1 (Build 65) software and presented with score-oriented dendrogram created by MALDI Biotyper MSP creation method (Bruker Daltonics, Bremen, Germany), where distance values are relative and routinely normalized to a maximum value of 1,000. Dendrograms were generated by similarity scoring of a set of mass spectra. The matching of unknown spectra to the main spectrum was evaluated based on dedicated score values and the results were reported as the best match to the Bruker database as per the manufacturer's manual [21].

\subsection{Detection of AHL Production}

AHL production in strain CSG20 was assayed by cross-streaking it perpendicular to C. violaceum CV026 on LB agar [22]. A purple pigmentation formed in C. violaceum CV026 suggests QS activity associated with the production of short-chain AHLs. E. carotovora GS101 and E. carotovora PNP22 were used as positive and negative controls, respectively [23].

\subsection{AHL Extraction}

AHL extraction from strain CSG20 was performed as reported previously [24,25]. In brief, the strain was grown overnight in LB medium $(15 \mathrm{~mL})$ buffered with $50 \mathrm{mM} 3$-[N-morpholino] propanesulfonic acid (MOPS) to $\mathrm{pH} 5.5$ to prevent spontaneous degradation of AHLs [26]. The culture supernatant was extracted twice with acidified ethyl acetate $(15 \mathrm{~mL}, 0.1 \% \mathrm{v} / \mathrm{v}$ glacial acetic acid) and evaporated to dryness under vacuum, resuspended in a minimal volume of acetonitrile and used for analysis in the high resolution triple quadrupole liquid chromatography (LC) tandem mass spectrometer (MS) to provide unequivocal confirmation of the presence of AHLs in strain CSG20. To identify AHLs via LC-MS/MS, we used synthetic AHLs, N-hexanoyl-L-homoserine lactone (C6-HSL) (obtained from Cayman Chemical, Ann Arbor, MI, USA) as standards and their spectra were compared to those of putative AHLs identified in strain CSG20. Stock solutions for standards $(1 \mathrm{mg} / \mathrm{mL})$ were prepared in acetonitrile and stored at $-20{ }^{\circ} \mathrm{C}$. 


\subsection{Triple Quadrupole Liquid Chromatography Mass Spectrometry (LC-MS/MS) Analysis}

LC was carried out on the Agilent 1290 Infinity LC system (Agilent Technologies Inc., Santa Clara, CA, USA) coupled with Agilent ZORBAX Rapid Resolution High Definition SB-C18 Threaded Column $(2.1 \mathrm{~mm} \times 50 \mathrm{~mm}, 1.8 \mu \mathrm{m}$ particle size $)$. The flow rate was set at $0.3 \mathrm{~mL} / \mathrm{min}\left(37^{\circ} \mathrm{C}\right)$ and the injection volume was fixed at $2 \mu \mathrm{L}$. Mobile phases A and B referred to $0.1 \% \mathrm{v} / \mathrm{v}$ formic acid in water and $0.1 \% \mathrm{v} / \mathrm{v}$ formic acid in acetonitrile, respectively. The gradient profile used was as follows (time: mobile phase A: mobile phase B): 0 min: 80:20, $7 \mathrm{~min}$ : 50:50, $12 \mathrm{~min}:$ 20:80, and $14 \mathrm{~min}:$ 80:20. MS detection from UHPLC separated compounds was performed on the Agilent 6490 Triple Quadrupole LC/MS system (Agilent Technologies Inc., Santa Clara, CA, USA). Precursor ion-scanning experiments were performed in positive ion mode with Q3 set to monitor for $m / z 102$ and Q1 set to scan a mass range of $\mathrm{m} / \mathrm{z} 80$ to $\mathrm{m} / \mathrm{z}$ 400. Molecular mass of $\mathrm{m} / \mathrm{z} 102$ refers to lactone ring thus indicating the presence of AHL. The LC/MS parameters were as follows: probe capillary voltage set at $3 \mathrm{kV}$, sheath gas at $11 \mathrm{~mL} / \mathrm{h}$, nebulizer pressure $20 \mathrm{psi}$, desolvation temperature at $200{ }^{\circ} \mathrm{C}$. The Agilent MassHunter software was used for the MS data analysis. Analysis was based on retention index and the comparison of EI mass spectra with standards.

\section{Results and Discussion}

Conventional methods for the identification and typing of bacteria are often tedious and time-consuming and frequently give inconclusive results for rare and atypical species. In contrast, proteomic characterization with the use of mass spectrometry can provide fast and reliable identification of microorganisms including multidrug resistant clinical pathogens [27,28]. Proteomic phenotypes from MALDI-TOF MS have been employed effectively for microbial biotyping and the BioTyperTM MALDI-TOF MS fingerprinting system allows researchers to identify a wide spectrum of bacteria, yeasts and fungi [29]. With the spectra generated from our strain CSG20, and processed by standard pattern matching with MALDI-TOF Biotyper software, we were able to identify it with confidence as K. pneumoniae (Figure 1). We believe, as others have forecasted, that this technology will soon be the method of choice for pathogen identification in the diagnostic laboratories.

Two major QS systems have been described in gram-negative bacteria, a type 1 system operating in intra-species communication and a type 2 system observed in inter-species communication. Type 1 QS is mediated by autoinducers known as AI-1 which are species-specific AHL's produced and regulated by AHL synthases encoded by the luxI gene [30,31]. The second protein in this system is the LuxR protein that detects AHL's and causes downstream change in gene expression [32] (Figure 2). Type 2 QS responds to a family of autoinducers known as AI-2 [33,34]. The AI-2 molecule is a furanosyl borate diester (Cao) produced by the LuxS protein encoded by the luxS gene [13,35]. Balestrino et al. [36] have reported the secretion of type 2 signaling molecules regulated by a homologue of the luxS gene, and the presence of AHL-lactonase, a quorum-quenching enzyme in K. pneumoniae [37-41]. De Araujo et al. [16] described the involvement of AI-2 autoinducers of K. pneumoniae in the regulation of biofilm formation. K. pneumonia strains producing AHLs have also been reported. Yin et al. [42] isolated them from the posterior dorsal surface of the tongue and demonstrated the production of 
$\mathrm{N}$-octanoylhomoserine lactone (C8-HSL) and $\mathrm{N}$-3-dodecanoylhomoserine lactone (C12-HSL) in these oral isolates. Our K. pneumoniae strain CSG20 produced C6-HSL (Figure 3).

Figure 1. Score-oriented dendrogram of clinical isolate K. pneumoniae strain CSG2.

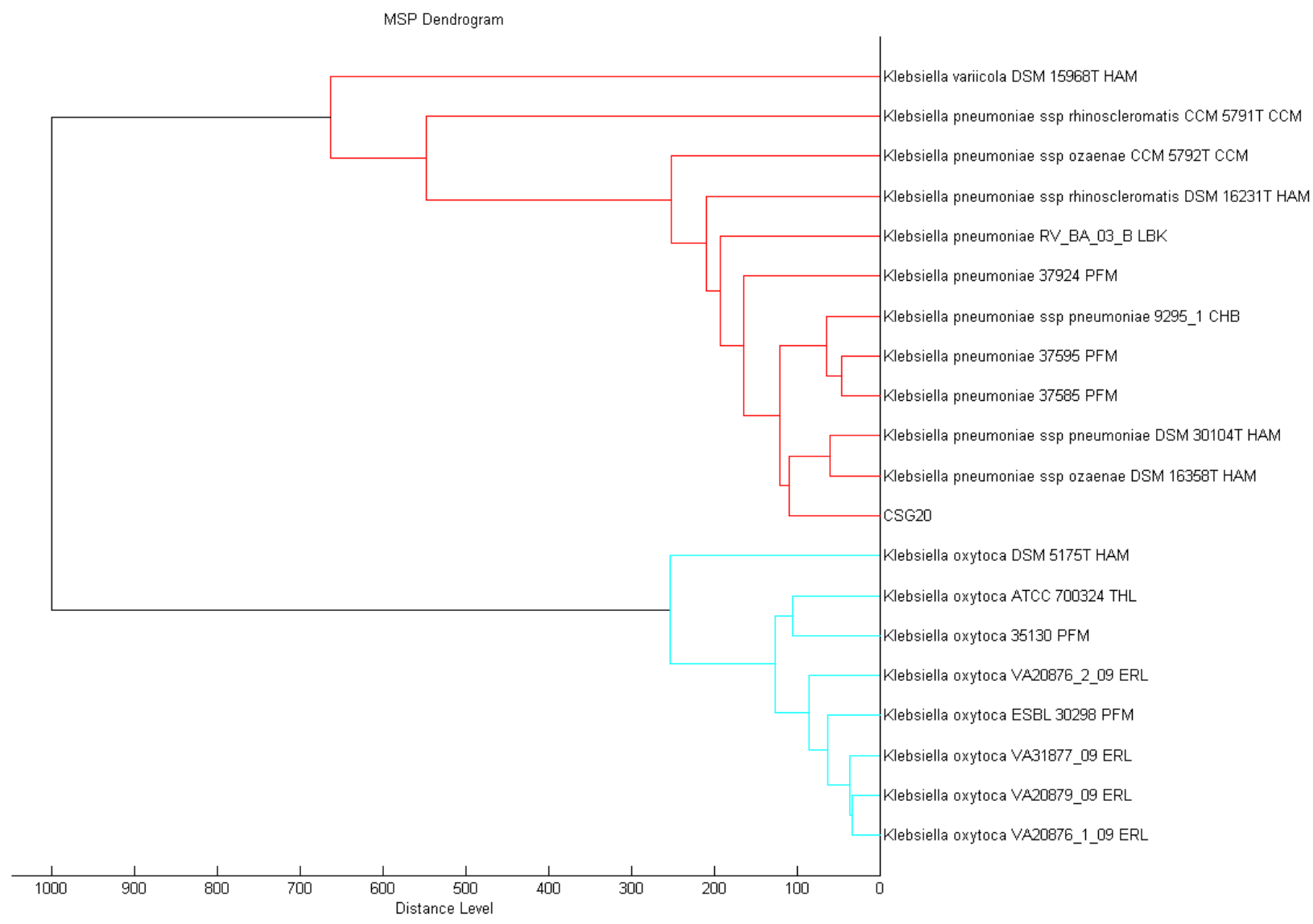

Figure 2. Diagram of quorum sensing.

\begin{tabular}{|l|l|}
\hline Low Density Population & High Density Population \\
\hline $\begin{array}{l}\text { Autoinducers } \\
\text { (egAHLs) }\end{array}$ & $\begin{array}{l}\text { QS autoinducer- } \\
\text { receptor complex }\end{array}$ \\
QS receptor \\
Regulate bacterial virulence, such as the \\
brofiltion of extracellular polysaccharides, \\
bactericidal activities
\end{tabular}


Figure 3. Mass spectra of AHLs extracted from K. pneumoniae strain CSG20 supernatant. (a) Total ion chromatogram of AHLs extract strain CSG20 and standard C6-HSL. (b) MS analysis of AHLs extract strain CGS20 shown product ion $\mathrm{m} / \mathrm{z} 101.90$ and precursor ion m/z 200.1 (Retention time: 1.970 min; Abundance: 439.96; Abundance \%: 7.07).

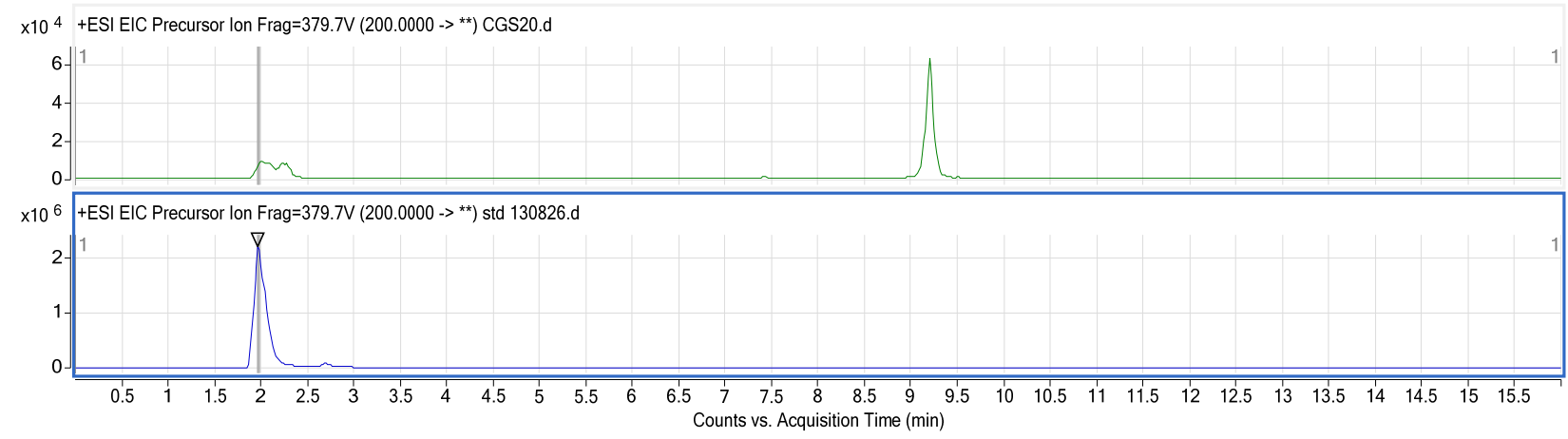

(a)

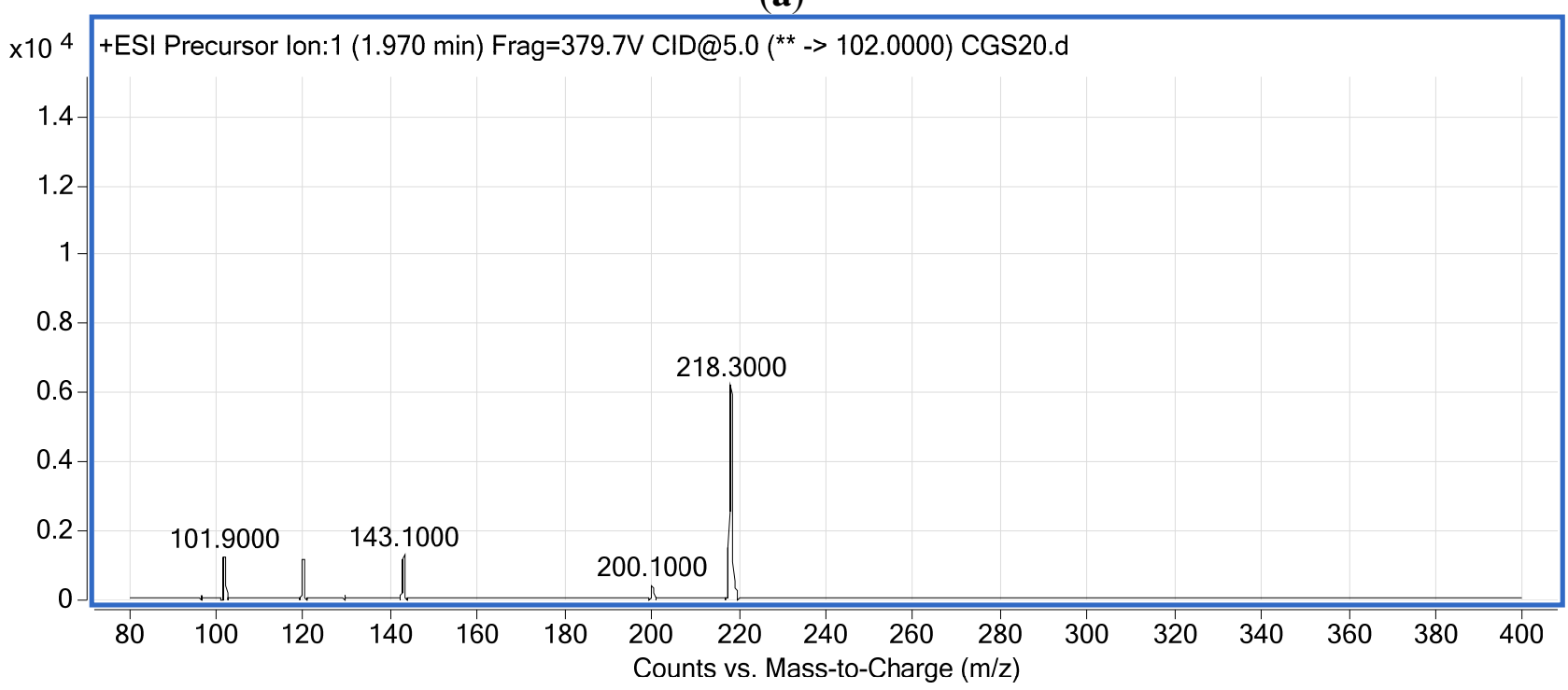

(b)

It is possible that strains from different sites of infection show different AHL production profiles, just as different growth conditions in the laboratory can affect the type and amount of AHLs produced in bacteria [43]. It has been demonstrated, for instance, that K. pneumoniae and K. oxytoca strains produced AHLs only when grown in LB medium in microaerophilic but not under aerobic conditions [43]. However, we were able to detect AHLs in our strain CSG20 that was grown aerobically. This could be due to our use of a $\mathrm{pH}$ buffer, MOPS, in the LB growth medium. By acidifying the growth medium, MOPS can prevent the inactivation of AHLs by lactonolysis that occurs in alkaline conditions [26]. It is possible that MOPS also stabilized the AHLs produced in our CSG20 strain.

The natural phenomenon of QS has been the focus of recent research towards the discovery of novel, non-antibiotic-dependent approaches to anti-infective therapy [44-50]. The description of new AHL signals in a multidrug resistant $K$. pneumoniae strain in this paper will provide additional molecular targets towards the quest for QS-based alternative treatment. 


\section{Conclusions}

Here, we were presented clinical isolate, K. pneumoniae strain CSG20 was produced C6-HSL. This funding will provide better understanding of QS activity of $K$. pneumoniae and may enable the anti-QS as one of the alternative anti-infective therapy.

\section{Acknowledgments}

This work was supported by two High Impact Research grants from the University of Malaya: (a) Bacteria Quorum Quenching HIR-MOHE Grant A000001-50001 awarded to Kok-Gan Chan; and (b) Nature Microbiome HIR-MOHE Grant UM.C/625/1/HIR/MOHE/CHAN/14/4, H50001-00-A000038 awarded to Yun Fong Ngeow; which are gratefully acknowledged.

\section{Conflict of Interest}

The authors declare no conflict of interest.

\section{References}

1. Podschun, R.; Ullmann, U. Klebsiella spp. as nosocomial pathogens: Epidemiology, taxonomy, typing methods, and pathogenicity factors. Clin. Microbiol. Rev. 1998, 11, 589-603.

2. Guentzel, M.N. Escherichia, Klebsiella, Enterobacter, Serratia, Citrobacter, and Proteus. In Medical Microbiology, 4th ed.; Galveston (TX): University of Texas Medical Branch at Galveston, Galveston, TX, USA, 1996.

3. Ko, W.C.; Paterson, D.L.; Sagnimeni, A.J.; Hansen, D.S.; von Gottberg, A.; Mohapatra, S.; Casellas, J.M.; Goossens, H.; Mulazimoglu, L.; Trenholme, G.; et al. Community-acquired Klebsiella pneumoniae bacteremia: Global differences in clinical patterns. Emerg. Infect. Dis. 2002, 8, 160-166.

4. McCloud, J.M.; Jameson, J.S. Life-threatening sepsis following treatment for haemorrhoids: A systematic review. Colorectal Dis. 2006, 8, 748-755.

5. Jong, G.M.; Hsiue, T.R.; Chen, C.R.; Chang, H.Y.; Chen, C.W. Rapidly fatal outcome of bacteremic Klebsiella pneumoniae pneumonia in alcoholics. Chest. J. 1995, 107, 214-217.

6. Fang, C.T.; Chuang, Y.P.; Shun, C.T.; Chang, S.C.; Wang, J.T. A novel virulence gene in Klebsiella pneumoniae strains causing primary liver abscess and septic metastatic complications. J. Exp. Med. 2004, 199, 697-705.

7. Lin, Y.T.; Jeng, Y.Y.; Chen, T.L.; Fung, C.P. Bacteremic community-acquired pneumonia due to Klebsiella pneumoniae: Clinical and microbiological characteristics in Taiwan, 2001-2008. BMC Infect. Dis. 2010, 10, 307.

8. Calfee, D.P. Crisis in hospital-acquired, healthcare-associated infections. Annu. Rev. Med. 2012, 63, 359-371.

9. Hirsch, E.B.; Tam, V.H. Detection and treatment options for Klebsiella pneumoniae carbapenemases (KPCs): An emerging cause of multidrug-resistant infection. J. Antimicrob. Chemother. 2010, 65, 1119-1125. 
10. Nordmann, P.; Poirel, L.; Toleman, M.A.; Walsh, T.R. Does broad-spectrum $\beta$-lactam resistance due to NDM-1 herald the end of the antibiotic era for treatment of infections caused by Gram-negative bacteria? J. Antimicrob. Chemother. 2011, 66, 689-692.

11. Paiva, J.A. Adding risk factors for potentially resistant pathogens, increasing antibiotic pressure and risk creating the "untreatable bacteria": Time to change direction. Intensiv. Care Med. 2013, 39, 779-781.

12. Sun, S.; Zhang, W.; Mannervik, B.; Andersson, D.I. Evolution of broad spectrum $\beta$-lactam resistance in an engineered metallo- $\beta$-lactamase. J. Biol. Chem. 2013, 288, 2314-2324.

13. Miller, M.B.; Bassler, B.L. Quorum sensing in bacteria. Annu. Rev. Microbiol. 2001, 55, 165-199.

14. Lindum, P.W.; Anthoni, U.; Christophersen, C.; Eberl, L.; Molin, S.; Givskov, M. $\mathrm{N}$-acyl-L-homoserine lactone autoinducers control production of an extracellular lipopeptide biosurfactant required for swarming motility of Serratia liquefaciens MG1. J. Bacteriol. 1998, 180, 6384-6388.

15. Coenye, T. Social interactions in the Burkholderia cepacia complex: Biofilms and quorum sensing. Future Microbiol. 2010, 5, 1087-1099.

16. De Araujo, C.; Balestrino, D.; Roth, L.; Charbonnel, N.; Forestier, C. Quorum sensing affects biofilm formation through lipopolysaccharde synthesis in Klebsiella pneumoniae. Res. Microbiol. 2010, 161, 595-603.

17. Dickschat, J.S. Quorum sensing and bacterial biofilms. Nat. Prod. Rep. 2010, 27, 343-369.

18. Kaufmann, G.F.; Sartorio, R.; Lee, S.H.; Rogers, C.J.; Meijler, M.M.; Moss, J.A.; Clapham, B.; Brogan, A.P.; Dickerson, T.J.; Janda, K.D. Revisiting quorum sensing: Discovery of additional chemical and biological functions for 3-oxo- $N$-acylhomoserine lactones. Proc. Natl. Acad. Sci. USA 2005, 102, 309-314.

19. McClean, K.H.; Winson, M.K.; Fish, L.; Taylor, A.; Chhabra, S.R.; Camara, M.; Daykin, M.; Lamb, J.H.; Swift, S.; Bycroft, B.W.; et al. Quorum sensing and Chromobacterium violaceum: Exploitation of violacein production and inhibition for the detection of $\mathrm{N}$-acylhomoserine lactones. Microbiology 1997, 143, 3703-3711.

20. Chen, J.W.; Koh, C.L.; Sam, C.K.; Yin, W.F.; Chan, K.G. Short chain N-acyl homoserine lactone production by soil isolate Burkholderia sp. strain A9. Sensors 2013, 13, 13217-13227.

21. MALDI Biotyper User Manual 2008, Version 20 SR1; Bruker Daltonic Inc.: Bremen, Germany, 2008.

22. Swift, S.; Karlyshev, A.V.; Fish, L.; Durant, E.L.; Winson, M.K.; Chhabra, S.R.; Williams, P.; Macintyre, S.; Stewart, G.S. Quorum sensing in Aeromonas hydrophila and Aeromonas salmonicida: Identification of the LuxRI homologs AhyRI and AsaRI and their cognate $\mathrm{N}$-acylhomoserine lactone signal molecules. J. Bacteriol. 1997, 179, 5271-5281.

23. McGowan, S.; Sebaihia, M.; Jones, S.; Yu, B.; Bainton, N.; Chan, P.; Bycroft, B.; Stewart, G.S.A.B.; Williams, P.; Salmond, G.P.C. Carbapenem antibiotic production in Erwinia carotovora is regulated by CarR, a homologue of the LuxR transcriptional activator. Microbiology 1995, 141, 541-550. 
24. Chan, K.G.; Atkinson, S.; Mathee, K.; Sam, C.K.; Chhabra, S.R.; Cámara, M.; Koh, C.L.; Williams, P. Characterization of $N$-acylhomoserine lactone-degrading bacteria associated with the Zingiber officinale (ginger) rhizosphere: Co-existence of quorum quenching and quorum sensing in Acinetobacter and Burkholderia. BMC Microbiol. 2011, 11, doi:10.1186/1471-2180-11-51.

25. Wong, C.S.; Yin, W.F.; Choo, Y.M.; Sam, C.K.; Koh, C.L.; Chan, K.G. Coexistence of quorum-quenching and quorum-sensing in tropical marine Pseudomonas aeruginosa strain MW3A. World J. Microbiol. Biotechnol. 2011, 28, 453-461.

26. Yates, E.A.; Philipp, B.; Buckley, C.; Atkinson, S.; Chhabra, S.R.; Sockett, R.E.; Goldner, M.; Dessaux, Y.; Camara, M.; Smith, H.; et al. $N$-acylhomoserine lactones undergo lactonolysis in a $\mathrm{pH}$-, temperature-, and acyl chain length-dependent manner during growth of Yersinia pseudotuberculoxis and Pseudomonas aeruginosa. Infect. Immun. 2002, 70, 5635-5645.

27. Maier, T.; Klepel, S.; Renner, U.; Kostrzewa, M. Fast and reliable maldi-tof ms-based microorganism identification. Nat. Methods 2006, doi:10.1038/nmeth870.

28. Spanu, T.; Posteraro, B.; Fiori, B.; D’Inzeo, T.; Campoli, S.; Ruggeri, A.; Tunmarello, M.; Canu, G.; Trecarichi. E.M.; Parisi, G.; et al. Direct MALDI-TOF mass spectrometry assay of blood culture broths for rapid identification of Candida species causing bloodstream infections: An observational study in two large microbiology laboratories. J. Clin. Microbiol. 2012, 50, 176-179.

29. Sogawa, K.; Watanabe, M.; Sato, K.; Segawa, S.; Ishii, C.; Miyabe, A.; Murata, S.; Saito, T.; Nomura, F. Use of the MALDI BioTyper system with MALDI-TOF mass spectrometry for rapid identification of microorganisms. Anal. Bioanal. Chem. 2011, 400, 1905-1911.

30. Fuqua, C.; Parcek, M.R.; Greenberg, E.P. Regulation of gene expression by cell-to-cell communication: Acyl-homoserine lactone quorum sensing. Annu. Rev. Genet. 2001, 35, 439-468.

31. Fuqua, C.; Greenberg, E.P. Listening in on bacteria: Acyl-homoserine lactone signalling. Nat. Rev. Mol. Cell Biol. 2002, 3, 685-695.

32. Voloshin, S.A.; Kaprelyants, A.S. Cell-cell interactions in bacterial populations. Biochem. (Mosc.) 2004, 69, 1268-1275.

33. Cloak, O.M.; Solow, B.T.; Briggs, C.E.; Chen, C.Y.; Fratamico, P.M. Quorum sensing and production of autoinducer-2 in Campylobacter spp., Escherichia coli O157:H7, and Salmonella enterica serovar Typhimurium in foods. Appl. Environ. Microbiol. 2002, 68, 4666-4671.

34. Federle, M.J.; Bassler, B.L. Interspecies communication in bacteria. J. Clin. Investig. 2003, 112, 1291-1299.

35. Cao, J.G.; Meighen, E.A. Purification and structural identification of an autoinducer for the luminescence system of Vibrio harveyi. J. Biol. Chem. 1989, 264, 21670-21676.

36. Balestrino, D.; Haagensen, J.A.; Rich, C.; Forestier, C. Characterization of type 2 quorum sensing in Klebsiella pneumoniae and relationship with biofilm formation. J. Bacteriol. 2005, 187, 2870-2880.

37. Dong, Y.H.; Gusti, A.R.; Zhang, Q.; Xu, J.L.; Zhang, L.H. Identification of quorum quenching $\mathrm{N}$-acyl homoserine lactonases from Bacillus species. Appl. Environ. Microbiol. 2002, 68, 1754-1759. 
38. Kim, M.H.; Choi, W.C.; Kang, H.O.; Lee, J.S.; Kang, B.S.; Kim, K.J.; Derewenda, Z.S.; Oh, T.K., Lee, C.H.; Lee, J.K. The molecular structure and catalytic mechanism of a quorum-quenching N-acyl-L-homoserine lactone hydrolase. Proc. Natl. Acad. Sci. USA 2005, 102, 17606-17611.

39. Dong, Y.H.; Wang, L.H.; Zhang, L.H. Quorum-quenching microbial infections: Mechanisms and implications. Philos. Trans. R. Soc. B 2007, 362, 1201-1211.

40. Chan, K.G.; Wong, C.S.; Yin, W.F.; Sam, C.K.; Koh, C.L. Rapid degradation of N-3-oxoacylhomoserine lactones by a Bacillus cereus isolate from Malaysian rainforest soil. Antonie van Leeuwenhoek 2010, 98, 299-305.

41. Hong, K.W.; Koh, C.L.; Sam, C.K.; Yin, W.F.; Chan, K.G. Quorum quenching revisited-From signal decay to signalling confusion. Sensors 2012, 12, 4661-4696.

42. Yin, W.F.; Purmal, K.; Chin, S.; Chan, X.Y.; Chan, K.G. Long chain $N$-acyl homoserine lactone production by Enterobacter sp. isolated from human tongue surfaces. Sensors 2012, 12, $14307-14314$.

43. Wang, H.; Cai, T.; Weng, M.; Zhou, J.; Cao, H.; Zhong, Z.; Zhu, J. Conditional production of acyl-homoserine lactone-type quorum-sensing signals in clinical isolates of enterobacteria. J. Med. Microbiol. 2006, 55, 1751-1753.

44. Adonizio, A.L.; Downum, K.; Bennett, B.C.; Mathee, K. Anti-quorum sensing activity of medicinal plants in southern Florida. J. Ethnopharmacol. 2006, 105, 427-435.

45. Chong, Y.M.; Yin, W.F.; Ho, C.Y.; Mustafa, M.R.; Hadi, A.H.A.; Awang, K.; Narrima, P.; Koh, C.L.; Appleton, D.R.; Chan, K.G. Malabaricone C from Myristica cinnamomea exhibits anti-quorum sensing activity. J. Nat. Prod. 2011, 74, 2261-2264.

46. Krishnan, T.; Yin, W.-F.; Chan, K.-G. Inhibition of quorum sensing-controlled virulence factor production in Pseudomonas aeruginosa PAO1 by Ayurveda spice clove (Syzygium Aromaticum) bud extract. Sensors 2012, 12, 4016-4030.

47. Tan, L.Y.; Yin, W.-F.; Chan, K.-G. Silencing quorum sensing through extracts of Melicope lunu-ankenda. Sensors 2012, 12, 4339-4351.

48. Koh, C.L.; Sam, C.K.; Yin, W.F.; Tan, L.Y.; Krishnan, T.; Chong, Y.M.; Chan, K.G. Plant-derived natural products as sources of anti-quorum sensing compounds. Sensors 2013, 13, 6217-6228.

49. Norizan, S.N.M.; Yin, W.F.; Chan, K.G. Caffeine as a potential quorum sensing inhibitor. Sensors 2013, 13, 5117-5129.

50. Tan, L.Y.; Yin, W.F.; Chan, K.G. Piper nigrum, Piper betle and Gnetum gnemon-natural food source with anti-quorum sensing properties. Sensors 2013, 13, 3975-3985.

(C) 2013 by the authors; licensee MDPI, Basel, Switzerland. This article is an open access article distributed under the terms and conditions of the Creative Commons Attribution license (http://creativecommons.org/licenses/by/3.0/). 